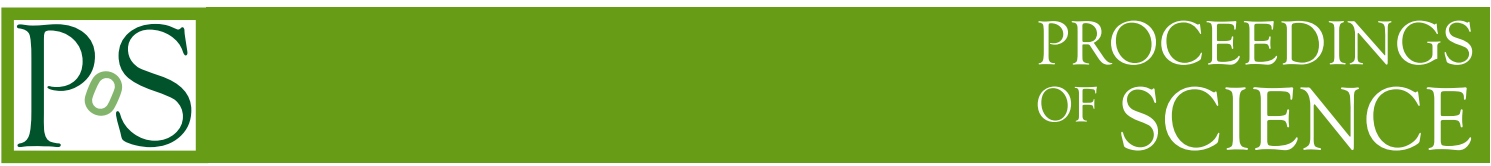

\title{
Observing alternatives to inflation
}

\author{
Patrick Peter* \\ $\mathscr{G} \mathbb{R} \mathbb{C} \mathscr{O}$ - Institut d'Astrophysique de Paris, UMR7095 CNRS \\ Université Pierre \& Marie Curie \\ 98 bis boulevard Arago \\ 75014 Paris, France \\ E-mail: petereiap.fr
}

We discuss the possibility that the inflationary paradigm, undoubtfully today's best framework to understand all the present cosmological data, may still have some viable challengers. The underlying idea for such discussions is that although inflation already passed quite a large number of tests, indeed enough to make it part of the so-called "standard model" of cosmology, it has always been thought indirect measurements: there is not a chance that we may ever directly check its validity, and therefore, in order to assert its factuality with increasing level of confidence, it is required that we compare its predictions not only to observations, but also to as many contenders as possible. Among other categories of possible models, we wish to put the emphasis in particular on bouncing cosmologies that, however not as complete as the inflation paradigm might be, could still represent a reasonnable way of explaining the current data. Hopefully, future data will be able to discriminate between these various sets of theories.

International Workshop on Cosmic Structure and Evolution - Cosmology2009,

September 23-25, 2009

Bielefeld, Germany

\footnotetext{
* Speaker.
} 


\section{Introduction}

Standard, pre-1980, cosmology, was plagued with many problems. Among those, most have received solutions in the framework of what eventually became known as the inflation paradigm [1-3]. Those problems were that of the existence of a singularity (not completely solved [4]), the question of why the properties of the cosmic microwave background (CMB) were the same over distances much larger than the horizon, and the fact that the Universe is observed to be flat [5] and homogeneous (this problem, although considerably alleviated by a phase of inflation, can be argued not to be actually solved by this phase) $[6,7]$. These are unavoidable difficulties coming directly from General Relativity (GR) and the Friedmann-Lemaître-Robertson-Walker (FLRW) metric whenever one assumes the Universe to be filled with a perfect fluid (dust or radiation in practice).

A different kind of problem is that of monopoles [10], namely particle-like topological defects [11] (TD) with large mass and magnetic interactions whose density in the early Universe can exceed by far the flatness limit, implying a very rapid crunch right after the big-bang, thus in disagreement with the very basic observation that the Universe merely exists! Also seemingly unavoidable, this problem relies heavily on the existence of a grand unification [12] for the interactions, unification which, although very seriously expected on theoretical grounds, is by no means as certain as GR and its FLRW solution. While solving these delicate matters, including the monopole excess problem, inflation provided, as an "unexpected" bonus, a means of calculating the expected spectrum of primordial perturbations $[8,9]$, providing a reasonable theory for the initial conditions of primordial perturbations.

Finally, there still is a set of cosmological puzzles which are not addressed within the inflationary paradigm. Those are the existence of dark matter [13], and the observation that the Universe is currently accelerating, which is interpreted as a yet-unknown constituent, called dark energy [14], assumed to be either a mere cosmological constant or a more elaborate quintessence field. Lastly, there is the very large asymmetry between matter and anti-matter that fits nowhere in the standard model of particle physics; this, however, may turn out to be more a problem of this latter field rather than of cosmology [15]. This last category is not solved by inflation, although there are models aiming at addressing those in unifying frameworks.

So the current situation is the following: inflation, as a paradigm, solves most of the cosmological puzzles in a consistent way, using GR and scalar fields (semi-)classically, and it can be implemented in high energy particle physics theories [16]. From the observational standpoint, it is fair to say that is also make falsifiable predictions (the spectrum for the density perturbations and, as a consequence, the CMB temperature anisotropy distribution), all these having been shown to be consistent with the data. It turned out, more recently, that is was also possible to implement an inflationary phase in a string [17] framework [18]. Inflation thus appears like a cosmological panacea, and one wonders why one would even consider alternatives.

The first reason to discuss noninflationary scenarios is quite simple: although inflation can be implemented in various theories, it is not the only possible cosmological outcome of these theories. Other scenarios turn out to be possible, not all of them being excluded by the data. Thus, there are challengers worth investigating. Besides, working out a challenger permits comparisons and different predictions. Future observations will then discriminate between models, as past observa- 
tions did: would it be merely to enforce the inflation paradigm, challengers would be already very useful.

Yet another reason to be concerned with noninflationary cosmology is the fact that it has problems of its own [19]. First of all, it does not address the question of the primordial singularity, although it is not clear that it is a question. More to the point are technical problems that need to be fixed. For instance, most models of inflation is heavily based on a fundamental scalar field ... yet no such field has ever been observed! This is a problem shared with many other theories, as in particular in particle physics the only missing bit is, precisely, the scalar Higgs particle. Some might also argue that using GR at energies that close to the Planck/string scale (the typical inflation scale is $E_{\text {inf }} \gtrsim 10^{-5} M_{\mathrm{Pl}}$, while the value of the scalar field responsible for the occurrence of inflation can even be larger than the Planck scale [20]) is questionable. Then comes a sort of hierarchy problem, by which one means the fact that at least one dimensionless number in the scalar field potential should be given a fine-tuned small value, of the order of $10^{-12}$; again, whether this is a negative point is arguable but should be kept in mind. Finally, one sets initial conditions at a time when the wavelength $\lambda=k^{-1} / a(t)[a(t)$ being the scale factor depending on time $t$, for a given comoving wavenumber $k$ ] of the perturbations can be smaller than the Planck length $\ell_{\mathrm{Pl}}$ itself; this is disturbing as one could imagine unknown and uncontrollable quantum gravity effects should be important, if not dominant, at such scales [21].

Having established the need for alternatives, the question remains as to which alternative? Old models based on TD (cosmic strings in particular) were found to be plausible, but only to partly [22] explain the large scale structure, i.e. at the $10 \%$ level (and then, some argue, with a slightly better fit). In that sense, TD cannot really be seen as an alternative.

Actual alternatives that claim to be compatible with the current data are not so many. The first, historically, called the Pre-big bang (PBB) [23], was based on string theory, using its dualities; the PBB is hard to reconcile with the data unless curvaton-like mechanism is taking place. Yet another mechanism, also involving string theory, was the so-called ekpyrotic scenario [24], which is subject to quite some controversy [25]. All these scenarios share the common feature of having a bouncing phase, at least in the Einstein frame. Therefore, it seems reasonable, as a generic alternative to inflationary cosmology, to consider bouncing cosmology, whose history is in fact much older than that of inflation, tracing back to the 1930's [26]! We shall not describes, in the short review below, models such as those based on string gas cosmology: they are as worth investigating as those presented below, but apart from the fact that space is lacking, I have never studied them in sufficient details to pretend discussing them here; I therefore refer the interested reader to Ref. [19] and the references given there for details (as well as another view on bounces and inflation caveats).

\section{Standard failures and some solutions}

The standard cosmological model is based on the FLRW metric [3]

$$
\mathrm{d} s^{2}=-\mathrm{d} t^{2}+a^{2}(t) \gamma_{i j} \mathrm{~d} x^{i} \mathrm{~d} x^{j}=a^{2}(\eta)\left(-\mathrm{d} \eta^{2}+\gamma_{i j} \mathrm{~d} x^{i} \mathrm{~d} x^{j}\right),
$$

where $\gamma_{i j}=\left(1+\frac{1}{4} \mathscr{K} \vec{x}^{2}\right)^{-1} \delta_{i j}$ is the spatial metric; Eq. (1.1) defines the conformal time $\eta$ as a function of the cosmic time $t$ and the scale factor $a$ through $\mathrm{d} t=a \mathrm{~d} \eta$. Plugging this form of the 
metric into Einstein equations with the stress-energy tensor for a perfect fluid with equation of state $w$, namely

$$
T_{\mu v}=(\rho+p) u_{\mu} u_{v}+p g_{\mu v}
$$

with energy density $\rho$, pressure $p=w \rho$ and $u_{\mu}$ is the fluid 4-velocity satisfying $u_{\mu} u^{\mu}=-1$, one finds that since $\nabla_{\mu} T^{\mu v}=0$ implies $\rho \propto a^{-3(1+w)}$, the solution for the scale factor reads (for $\mathscr{K}=0$ )

$$
a \propto t^{2 /[3(1+w)]} \propto \eta^{2 /(1+3 w)},
$$

provided $w \neq-1$. For a dust-dominated Universe with $w=0$, this is $a_{\text {dust }} \sim t^{2 / 3} \sim \eta^{2}$, while the radiation-dominated case $w=\frac{1}{3}$ is $a_{\mathrm{rad}} \sim t^{1 / 2} \sim \eta$ : in both cases, the limit when $t, \eta \rightarrow 0$ is singular, namely $a \rightarrow 0$. That is the origin of the singularity problem. In a bouncing cosmology, one assumes that, for whatever reason to be determined afterwards, the scale factor is bounded from below, so that, at all times, $a \neq 0$. As a result, the singularity problem is merely a non issue in the bouncing case ... but then of course, the question remains of the matter content which permits such a scale factor behavior.

The horizon problem is slightly more involved, although not that much [27]. To recall, the particle horizon at a point $P$ (the observer, say) is that surface dividing all points in the Universe into two distinct families, namely those that have already been observed at time $t$ by $P$, and those that have not. Specifically, integrating along the past light-cone at the point $P$, all the way to the origins of time $t_{\mathrm{i}}$, one finds a particle horizon exists provided the integral

$$
d_{\mathrm{H}}=a(t) \int_{t_{\mathrm{i}}}^{t} \frac{\mathrm{d} \tau}{a(\tau)}
$$

converges. The quantity $d_{\mathrm{H}}$ is called the horizon, and the problem in standard cosmology is that not only is it finite (the integral converges for a perfect fluid as before provided $w>-\frac{1}{3}$ ), but also, in comparison to the Hubble scale, it is small. In the framework of a bouncing Universe, one assumes that there is no such thing as the origin of time, and hence one sends $t_{\mathrm{i}}$ to negative infinity. If, during the contracting phase, one also have domination by a perfect fluid with equation of state $w>-\frac{1}{3}$, then the integral diverges, and so it remains infinite for all subsequent times, whatever happens at the bounce: all points in the Universe have, at some stage, been in causal contact.

The flatness problem now mostly stems from the equation which expresses the density $\rho(t)$ relative to the critical density $\rho_{\mathrm{c}}$. With $H \equiv \dot{a} / a$ (a dot meaning $\mathrm{d} / \mathrm{d} t$ ), this is

$$
\frac{\mathrm{d}}{\mathrm{d} t}|\Omega-1|=-2 \frac{\ddot{a}}{\dot{a}^{3}}, \quad \Omega \equiv \frac{\rho(t)}{\rho_{\mathrm{c}}(t)}=\frac{8 \pi G_{\mathrm{N}}}{3 H^{2}(t)} \rho(t) .
$$

For a nonaccelerating ( $\ddot{a} \geq 0$ ) expansion ( $\dot{a}>0$ ), it is clear that $\Omega$ is always moving away from unity: the flat Universe $(\Omega=1)$ is unstable. Inflation solves this problem by having a phase of accelerated expansion ... the bouncing paradigm suggests just the opposite, namely that $|\Omega-1|$ decreases because of a nonaccelerated contraction that lasted sufficiently long to compensate for the currently ongoing expansion.

With homogeneity, the situation is more intricate. Inflation proposes a dynamical mechanism by which any pre-existing inhomogeneity and anisotropy is washed out: an extremely small homogeneous region is almost instantaneously expanded into a size much larger than the current Hubble 
scale. Although this tremendously alleviates the problem, it remains to assume that there was a sufficiently homogeneous region of the required size to begin with [6] (see however Ref. [7] for a counter-argument). Whether this purely classical requirement is actually necessary may be still debatable, it is however not immediately obvious what this implies at the quantum level since homogeneous regions presumably do have a non-negligible probability to occur in the first place in a (yet-unknown) quantum gravity setup. For the bouncing situation, more information on the initial condition is needed.

At the origin of the contracting phase, the Universe is supposed to be large and extremely dilute, so both the stress-energy and the Ricci tensor are, by virtue of Einstein equations, very small. Adding the requirement that the Weyl tensor should also be small (i.e. we assume the Weyl curvature hypothesis [28]), we ensure the geometry to be almost flat to begin with, and look at the growth of whatever initial inhomogeneities we have have started with.

Since we assume a rarefied Universe, any inhomogeneity, even a large one having $\delta \rho / \rho \gtrsim 1$, has negligible self-gravitation because $\rho$ is small. In much the same way as it is true that sound waves do not condense, these initial inhomogeneities must dissipate. Let us now restrict attention to the (relevant) dust-dominated contraction for which the Jeans length is $\lambda_{\mathrm{J}}=c_{\mathrm{S}} \sqrt{\pi /\left(G_{\mathrm{N}} \rho\right)} \propto$ $a^{3 / 2}$, with $c_{\mathrm{S}}$ the sound velocity, which in this case is essentially the equation of state parameter $c_{\mathrm{S}} \sim w \ll 1$ : we cannot at this level approximate $w \simeq 0$ as this would imply a vanishing Jeans length and hence exponential growth of all scales! in fact, provided $w \neq 0$, which it cannot strictly be since there will always, in practice, be some amount, however tiny, of interaction between particles, it is always to set the initial sufficiently backwards in time to have the scale factor, hence the Jeans length, as large as one wants. We thus assume $\lambda_{\mathrm{J}}$ to be larger than any scale than any scale of cosmological relevance today.

The dust velocity evolving as $v \propto a^{-1}$ and the number density as $n \propto a^{-3}$, the mean free path is $\lambda_{\text {MFP }}=(n \sigma)^{-1} \propto a^{3}$, with $\sigma$ the interaction cross-section between dust particles. Again, although $\sigma$ is expected to be small for the dust approximation to be valid, it cannot vanish entirely. For a given wavelength $\lambda$, the associated dissipation time is

$$
t_{\mathrm{d}}(\lambda)=\frac{\lambda}{v}\left(1+\frac{\lambda}{\lambda_{\mathrm{MFP}}}\right)
$$

which should be compared with the Hubble time $t_{\mathrm{H}}$.

The dust scale factor relates with the Hubble scale $R_{\mathrm{H}}$ through $a \propto R_{\mathrm{H}}^{2 / 3}$, and hence $\lambda_{\text {MFP }}=$ $R_{\mathrm{H}}^{2} / L$, with $L$ an unknown constant with the dimension of a length, in principle to be determined by the microphysics at the time at which we want to fix the initial conditions. Taking the wavelength to be a fixed fraction $x$ of the Hubble scale now $R_{0}$, i.e. $\lambda=x R_{0}$, and similarly the Hubble radius at the time considered to be $R_{\mathrm{H}}=y R_{0}$, one finds that the ratio of the dissipation to the Hubble times is

$$
\frac{t_{\mathrm{d}}(\lambda)}{R_{\mathrm{H}}} \propto x y^{-1 / 3}\left(1+\frac{L}{R_{0}} \frac{x}{y^{2}}\right)
$$

so that it suffices to take initial conditions sufficiently far in the past when the Universe was much larger than it is now, i.e., to take $y \gg 1$ (with $x$ of order unity and $L$ of order the present day Hubble scale), to find $t_{\mathrm{d}} \ll R_{\mathrm{H}}$ : in a characteristic Hubble time $R_{\mathrm{H}}$, the system has had plenty of characteristic dissipation times, and so has dissipated completely before gravity begun to play any 
role. Actually, provided the Universe spends a sufficiently long time in this contracting phase, the diffusion is so effective that only quantum fluctuations due to the uncertainty principle could survive: vacuum initial conditions for the perturbations is a consequence of the homogenisation mechanism.

\section{Modelling the bounce}

GR with ordinary fluids does not admit bounce solutions. Indeed, the metric (1.1) with the fluid (1.2) provide the Einstein (Friedmann) equations (in the absence of cosmological constant)

$$
\left(\frac{a^{\prime}}{a}\right)^{2}+\mathscr{K}=\frac{8 \pi G_{\mathrm{N}}}{3} a^{2} \rho \quad \text { and } \quad \frac{a^{\prime \prime}}{a}=\frac{8 \pi G_{\mathrm{N}}}{6} a^{2}(\rho-3 P)-\mathscr{K},
$$

where $^{\prime} \equiv \mathrm{d} / \mathrm{d} \eta$. A bounce being defined as the point at which the scale factor reaches a minimum value, it means one must have, at the bounce conformal time, $a_{\mathrm{b}}^{\prime}=0$ and $a_{\mathrm{b}}^{\prime \prime}>0$. Unless $\mathscr{K}>0$, the first condition requires that the energy density for the matter vanishes at that time. It is therefore not surprising that most classical bouncing models relies on closed spatial sections [29]. Other kinds of models either contain negative energies [30] (and can therefore only by analyzed as effective) or contain nonstandard terms [31]. Although all of these models have their specific interests, I would like to concentrate on yet another category, based on quantum cosmology [32].

The model thanks to which one manages to perform a bounce and produce a scale invariant spectrum of scalar perturbations (see the next section) turns out to be the simplest possible one, namely one for which one gravity plus a single perfect fluid with almost vanishing equation of state. When quantum cosmological effects are taken into account, it is no longer necessary to demand positive spatial curvature, and therefore we can set $\mathscr{K}=0$.

In quantum cosmology, separating the background and the perturbations means factorizing the Universe wavefunction $\psi$ into zeroth and second orders as

$$
\psi=\psi^{(0)}(a, \eta) \times \psi^{(2)}\left[a, \Psi(\vec{x}), \Phi(\vec{x}), h_{i j}(\vec{x}), \eta\right] .
$$

The idea is to solve the Wheeler-de Witt equation for the background wavefunction first, and using a Bohmian interpretation [33], derive the quantum trajectory for the scale factor. One finds, for the scale factor

$$
a(\tau)=a_{0}\left[1+\left(\frac{\tau}{\tau_{0}}\right)^{2}\right]^{1 /[3(1-w)]} \quad \text { with } \quad \mathrm{d} \eta=[a(\tau)]^{3 w-1} \mathrm{~d} \tau,
$$

where $a_{0}$ and $\tau_{0}$ are two arbitrary integration constants. They represent respectively the minimum value of the scale factor and the bounce duration. In terms of such a solution, one now needs to consider metric perturbations. This we do in the next section.

\section{Spectrum of perturbations}

In section 1, we showed that the initial conditions for the primordial fluctuations should be vacuum like. With this in mind, we shall now propagate these perturbations through the bounce itself in order to determine the observational predictions of the bouncing paradigm. 
The metric, one step beyond the background level described above, now reads

$$
\mathrm{d} s^{2}=a^{2}(\eta)\left\{-(1+2 \Phi) \mathrm{d} \eta^{2}+\left[\gamma_{i j}(1-2 \Psi)+h_{i j}\right] \mathrm{d} x^{i} \mathrm{~d} x^{j}\right\}
$$

in the longitudinal gauge (we do not consider here the possible vector part). The tensor $h_{i j}=h_{j i}$ is divergenceless $\left(\nabla_{i} h^{i j}=0\right)$ and traceless $\left(\gamma^{i j} h_{i j}=0\right)$; spatial indices are raised and lowered with the background spatial metric $\gamma_{i j}$. As for the perturbed stress-energy tensor components, they are

$$
\delta T_{00}=\rho a^{2}\left(\frac{\delta \rho}{\rho}+2 \Phi\right), \quad \delta T_{0 i}=-\rho a^{2}(1+w) \nabla_{i} v, \quad \delta T_{i j}=P a^{2}\left(h_{i j}-2 \gamma_{i j} \Psi+\frac{\delta p}{p} \gamma_{i j}+\pi_{i j}\right),
$$

where the anisotropic stress tensor $\pi_{i j}$ can be further decomposed into scalar and tensor part through $\pi_{i j}=\left(\nabla_{i} \nabla_{j}-\frac{1}{3} \gamma_{i j} \Delta\right) \bar{\pi}+\bar{\pi}_{i j}$, the latter part $\bar{\pi}_{i j}$ being divergenceless and traceless.

We have not considered vector modes: those, as is well known, are not really dynamical and, in an expanding Universe, are rapidly washed out if no anisotropic stress is present to begin with. In a contracting Universe, the situation is far from being that clear, as one would for instance expect those to grow (in practice with the square of the inverse scale factor), thus potentially breaking the perturbation approximation, thereby destroying the whole picture as a viable cosmological model [34]. However, such a dramatic effect depends not only on the specific details of the contracting and bouncing phases, but also on the initial conditions which, as discussed above, may simply imply the vector modes to identically vanish. This is what we assume in what follows.

Let us now come back to the quantum cosmology situation depicted in the previous section and plug the solution (2.3) into the equation for the second order part of the wavefunction (note that in this very simple case, one also has that the two potentials appearing in the metric expansion reduce to a single one, namely $\Phi=\Psi$ ). Fourier expanding in terms of the comoving wavenumber $k$, one finds the usual mode equation for the Mukhanov-Sasaki variable $v$, related to the Bardeen potential $\Phi$ through

$$
\Delta \Phi=-\frac{3}{2} a \ell_{\mathrm{PI}}^{2} \sqrt{\frac{\rho+p}{w}} \frac{\mathrm{d}}{\mathrm{d} \eta}\left(\frac{v}{a}\right)
$$

namely

$$
v_{k}^{\prime \prime}+\left(c_{\mathrm{s}}^{2} k^{2}-\frac{a^{\prime \prime}}{a}\right) v_{k}=0,
$$

where the sound velocity $c_{\mathrm{s}}=\sqrt{w} \ll 1$ cannot vanish. During the contracting phase, the "potential" $V=a^{\prime \prime} / a$ of this Schrödinger-like equation is important close to the bounce: for sufficiently large negative $\eta=\eta_{\text {ini }}, V \ll c_{\mathrm{s}}^{2} k^{2}$, and one can impose vacuum initial conditions, namely set

$$
v_{k}\left(\eta<\eta_{\text {ini }}\right)=\frac{\mathrm{e}^{-i c_{\mathrm{s}} k \eta}}{\sqrt{2 c_{\mathrm{s}} k}}
$$

and subsequently evolve this function with Eq. (3.4). The scalar spectrum $\mathscr{P}_{\Phi} \propto k^{3}\left|\Phi_{k}\right|^{2} \propto A_{\mathrm{s}}^{2} k^{n_{\mathrm{S}}-1}$ is then obtained once $\Phi$, reconstructed from $v$, reaches, in the expanding phase, a constant value: this is the dominant mode.

The spectrum for the gravitational wave (tensor mode) is obtained in a completely similar way. In fact, expanding $h_{i j}$ on a basis on time-independent polarisations modulated by an amplitude 
$\mu=h / a$, and going to Fourier space, one finds

$$
\mu_{k}^{\prime \prime}+\left(k^{2}-\frac{a^{\prime \prime}}{a}\right) \mu_{k}=0
$$

which, apart from the unimportant factor of the sound speed, is the same as Eq. (3.4). The initial condition can be set in an exactly similar way, so as the dynamics is also the same, one expects that the tensor spectrum $\mathscr{P}_{h} \propto k^{3}\left|h_{k}\right|^{2} \propto A_{\mathrm{T}}^{2} k^{n_{\mathrm{T}}}$ will be, up to a normalisation factor, equal to the scalar spectrum.

The actual solution which we found, either by numerically integrating the mode equation or by making approximations and matchings, is that the spectral indices satisfy

$$
n_{\mathrm{T}}=n_{\mathrm{S}}-1=\frac{12 w}{1+3 w} \ll 1 .
$$

Normalizing to the actual data, i.e. setting $A_{\mathrm{T}}^{2}=2.08 \times 10^{-10}$, one can then calculate the unknown parameters $a_{0}$ and $\tau_{0}$. Those provide the curvature at the bounce itself, and we find

$$
\tau_{0} a_{0}^{3 w} \sim 10^{3} \ell_{\mathrm{Pl}}
$$

which turns out to be precisely the length scale at which one expects quantum gravity corrections to become important, while the full quantum theory is still unnecessary: the Wheeler-de Witt equation is supposed to make sense in this regime, and our result is robust.

In this category of models, one also finds that the tensor-to-scalar ratio is related to the scalar spectral index, although in a different way from the usual inflation case: we find $(T / S) \simeq 4 \times$ $10^{-2} \sqrt{n_{\mathrm{S}}-1}$. This result is to be compared with the so-called consistency relation in single field inflation, which essentially states that the ratio $(T / S)$ ought to be proportional to $n_{\mathrm{S}}-1$ and not its square root. This, together with the fact that $n_{\mathrm{S}}>1$, again in contradiction with the inflation prediction, provides another way of testing either inflation or one of its contenders.

This kind of models is, of course, not without drawbacks. In particular, the minimum value of the scale factor, for instance, is extremely large, $a_{0} \gtrsim 10^{20} \ell_{\mathrm{Pl}}$, which implies a conceptual problem as a number of this order of magnitude is rather unexpected, and in fact the probability, calculated from the wavefunction, for the occurrence of such a large value, is more than tiny (of the order of $\mathrm{e}^{-10^{89}}$ in extreme cases!). Generalized solution for the background wavefunction, describing a moving Gaussian with nonvanishing velocity term along the $a$-axis, provide a means of solving such problems [35].

\section{Conclusions}

I have presented a very rapid tour of the reasons why it is still possible to find consistent cosmological models that do not include a phase of inflation, insisting in particular on the simplest possibility, sticking to 4 dimensional GR-based (and its quantum version) theories. Other, more involved, models are possible, and many share with the above most of their characteristic features. Others still exhibit different properties such as oscillations in the predicted spectrum of primordial perturbations. Increased precision experiments such as Planck, whose first scientific data are 
expected very soon, will be hopefully able to discriminate between the various theories discussed here and the more standard inflation paradigm.

Concentrating on a very specific model based on the Wheeler-de Witt equation, using a Bohmian interpretation for the quantum, I have summarized the results obtained through the years that tend to show that plain GR plus a perfect field can produce a sound model of the early Universe. Of course, this model is most definitely oversimplified. However, it has the advantage to be able to reproduce most of the data without introducing arbitrary scalar fields. Although clearly not perfect and far too simple as yet, it exemplifies the fact that alternatives are still viable and deserve further investigations.

Further work is necessary in order to assert whether such models can be made more than viable, but actually competitive alternatives. Like inflation models, most bouncing scenarios have been developed within the framework of a single fluid/field component; as the Universe contains more than one such component, the very first generalisation one needs to implement is that in which both matter and radiation (and even perhaps a cosmological constant) are present at the same time. In brief, one should try and build a scenario that really accounts for all observations. And as far as future data are concerned, for instance, it is of paramount interest to understand what is the amount of nongaussianity they tend to produce, and in what configurations. Again, this will have to be compared with generic inflationary predictions, and then with observations, whenever available.

\section{Acknowledgments}

I wish to thank the organizers of the meeting that turned out to be extremely fruitful and interesting. Moreover, I want to warmly thank all my collaborators on the topics I reviewed here, namely R. Abramo, J. Fabris, F. Falciano, M. Lilley, J. Martin, E. Pinho, N. Pinto-Neto, D. Schwarz and I. Yasuda. I wish to thank especially J. Martin for his careful reading of the manuscript.

\section{References}

[1] A. Linde, Particle Physics and Inflationary Cosmology, (Harwood, 1990).

[2] M. Lemoine, J. Martin and P. Peter (Eds.), Inflationary Cosmology, Lect. Notes Phys. 738 (Springer, Heidelberg, 2008).

[3] P. Peter and J.-P. Uzan, Primordial Cosmology (Oxford University Press, UK, 2009).

[4] A. Borde, A. A. Guth and A. Vilenkin, Inflationary spacetimes are not past-complete, Phys. Rev. Lett. 90, 151301 (2003) [gr-qc/ 0110012 ]; A. Borde and A. Vilenkin, Singularities in Inflationary Cosmology: A Review, Int. J. Mod. Phys. D5, 813 (1996) [gr-qc/9612036]; Eternal inflation and the initial singularity, Phys. Rev. Lett. 72, 3305 (1994) [gr-qc/9312022].

[5] A. H. Guth, Inflationary universe: A possible solution to the horizon and flatness problems, Phys. Rev. D23, 347 (1981).

[6] N. Deruelle and D. S. Goldwirth, Conditions for inflation in an initially inhomogeneous universe, Phys. Rev. D51, 1563 (1995) [arXiv: gr-qc/ 9409056 ] E. Calzetta and M. Sakellariadou, Semiclassical effects and the onset of inflation, Phys. Rev. D47, 3184 (1993) [arXiv: gr-qc/9209007]; Inflation in inhomogeneous cosmology, Phys. Rev. D45, 2802 (1992); D. S. Goldwirth and T. Piran, Initial conditions for inflation, Phys. Rep. 214, 223 (1992). 
[7] H. Kurki-Suonio, P. Laguna and R. A. Matzner, Inhomogeneous inflation: numerical evolution, Phys. Rev. D48, 3611 (1993) [arXiv: astro-ph/9306009].

[8] V. F. Mukhanov and G. V. Chibisov, Quantum fluctuations and a nonsingular universe, JETP Lett. 33, 532 (1981).

[9] V. F. Mukhanov, H. A. Feldman, and R. H. Brandenberger, Theory of cosmological perturbations, Phys. Rep. 215, 203 (1992).

[10] J. P. Preskill, Cosmological Production of Superheavy Magnetic Monopoles, Phys. Rev. Lett. 43, 1365 (1979).

[11] A. Vilenkin and E. P. S. Shellard, Cosmic Strings and Other Topological Defects (Cambridge University Press, 2000).

[12] P. Langacker, Grand Unified Theories and Proton Decay, Phys. Rep. 79, 1 (1981).

[13] G. Bertone, D. Hooper and J. Silk, Particle Dark Matter: Evidence, Candidates and Constraints, Phys. Rep. 405, 279 (2005) [hep-ph/ 0404175$].$

[14] J. Martin, Quintessence: a mini-review, Mod. Phys. Lett. A23, 1252 (2008) [astro-ph/ 0803.4076$]$; E. J. Copeland, M. Sami, S. Tsujikawa, Dynamics of dark energy, Int. J. Mod. Phys. D15, 1753 (2006) [hep-th/ 0603057$].$

[15] See e.g. J. Kang, P. Langacker, T. Li and T. Liu, Electroweak baryogenesis, CDM and anomaly-free supersymmetric $U(1)$ models, [hep-ph/0911.2939] and references therein.

[16] D. Baumann, TASI Lectures on Inflation (2009) [hep-ph/0907. 5424]; D. H. Lyth and A. Riotto, Particle physics models of inflation and the cosmological density perturbation, Phys.Rep. 314, 1 (1999) [hep-ph/9807278].

[17] K. Becker, M. Becker and J. H. Schwarz, String theory and M-theory (Cambridge University Press, UK, 2007); B. Zwiebach, A first course in string theory (Cambridge University Press, 2004); J. Polchinski, String theory (Cambridge University Press, 2001), among many others.

[18] D. Baumann and L. McAllister, Advances in Inflation in String Theory, Annu. Rev. Nuc. Part. Sci, to appear (2009) [hep-th/ 0901.0265$]$.

[19] R. H. Brandenberger, Alternatives to cosmological inflation, CosPA08 proceedings (2009) [hep-th/0902 . 4731]; Conceptual problems of inflationary cosmology and a new approach to cosmological structure formation, in Ref. [2], p. 393 (2008).

[20] D. H. Lyth, Particle physics models of inflation, in Ref. [2], p. 81 (2008) [arXiv: hep-th/0702128] and references therein.

[21] J. Martin, Inflationary cosmological perturbations of quantum-mechanical origin, Lect. Notes Phys. 669, 199 (2005) [hep-th/ 0406011 ], see in particular last section and references.

[22] A. A. Fraisse, C. Ringeval, D. N. Spergel, F. R. Bouchet, Small-angle CMB temperature anisotropies induced by cosmic strings, Phys. Rev. D78, 043535 (2008) [astro-ph/0708.1162]; N. Bevis, M. Hindmarsh, M. Kunz and J. Urrestilla, Fitting CMB data with cosmic strings and inflation, Phys. Rev. Lett. 100021301 (2008) [astro-ph/ 0702223 ]; A. A. Fraisse, Constraints on topological defects energy density from first year WMAP results (2005) [ast ro-ph/ 05034 02]; F. R. Bouchet, P. Peter, A. Riazuelo, M. Sakellariadou, Evidence against or for topological defects in the BOOMERanG data?, Phys. Rev. D65, 021301 (2002) [astro-ph/ 0005022 ]. 
[23] M. Gasperini and G. Veneziano, String theory and pre-big bang cosmology, in "Beyond the Big Bang", ed. by Ruediger Vaas (Springer-Verlag, 2007) [hep-th/0 703055]; M. Gasperini and G. Veneziano, The pre-big bang scenario in string cosmology, Phys. Rep. 373, 1 (2003).

[24] J.-L. Lehners, P. J. Steinhardt and N.Turok, The return of the Phoenix Universe, Gravity Research Foundation essay (2009) [hep-th/0910.0834]; J. Khoury, B. A. Ovrut, P. J. Steinhardt and N. Turok, The ekpyrotic universe: Colliding branes and the origin of the hot big bang, Phys. Rev. D64, 123522 (2001) [hep-th/0103239].

[25] R. Kallosh, J. U Kang, A. Linde and V. Mukhanov, The new ekpyrotic ghost, JCAP 0804, 018 (2008) [hep-th/0712.2040; J. Martin, P. Peter, N. Pinto-Neto and D. J. Schwarz, Passing through the bounce in the Ekpyrotic models, Phys. Rev. D65, 123513 (2002) [hep-th/ 0204227 ]; Comment on Density perturbations in the ekpyrotic scenario, Phys. Rev. D67, 028301 (2003) [hep-th/0204222]; R. Kallosh, L. Kofman and A. Linde, Pyrotechnic Universe, Phys. Rev. D64, 123523 (2001) [hep-th/ 0104073 ].

[26] R. C. Tolman, On the theoretical requirements for a periodic behaviour of the Universe, Phys. Rev. 38, 1758 (1931); G. Lemaître, L'Univers en expansion, Ann. Soc. Sci. Bruxelles (1933);

A. A. Starobinsky, On one non-singular isotropic cosmological model, Sov. Astron. Lett.4, 82 (1978); R. Durrer and J. Laukerman, The oscillating Universe: an alternative to inflation, Class. Quantum Grav. 13, 1069 (1996).

[27] P. Peter and N. Pinto-Neto, Cosmology without inflation, Phys. Rev. D78, 063504 (2008) [gr-qc/0809.2022].

[28] R. Penrose, in General Relativity: An Einstein Centenary Survey, S. W. Hawking and W. Israel (Eds.) (Cambridge University Press, Cambridge, England, 1979), p. 581; N. Pelavas and K. Lake, Measures of gravitational entropy I. Self-similar spacetimes, Phys.Rev. D62, 044009 (2000) [gr-qc/9811085].

[29] F. T. Falciano, M. Lilley and P. Peter, A classical bounce: constraints and consequences, Phys. Rev. D77, 083513 (2008) [gr-qc/0802.1196]; J. Martin and P. Peter, On the properties of the transition matrix in bouncing cosmologies, Phys. Rev. D69, 107301 (2004) [hep-th/ 0403173 ]; On the "causality argument" in bouncing cosmologies, Phys. Rev. Lett. 92, 061301 (2004) [astro-ph/0312488]; Parametric amplification of metric fluctuations through a bouncing phase, Phys. Rev. D68, 103517 (2003) [hep-th/ 0307077 ].

[30] F. Finelli, P. Peter and N. Pinto-Neto, Spectra of primordial fluctuations in two-perfect-fluid regular bounces, Phys. Rev. D77, 103508 (2008) [gr-qc/0709.3074 ]; V. Bozza, G. Veneziano, Regular two-component bouncing cosmologies and perturbations therein, JCAP 0509, 007 (2005) [gr-qc/ 0506040$]$; Scalar perturbations in regular two-component bouncing cosmologies, Phys. Lett. B625, 177 (2005) [hep-th/ 05020 47]; L. E. Allen and D. Wands, Cosmological perturbations through a simple bounce, Phys. Rev. D70, 063515 (2004) [astro-ph/ 0404441 ]; P. Peter and N. Pinto-Neto, Primordial perturbations in a non singular bouncing universe model, Phys. Rev. D66, 063509 (2002) [hep-th/ 0203013$].$

[31] L. R. Abramo, P. Peter and I. Yasuda, Non singular bounce in modified gravity [hep-th/0910.3422]; L. R. Abramo and P. Peter, $\mathscr{K}$-bounce, JCAP 0709, 001 (2007) [astro-ph/0705.2893].

[32] P. Peter, E. J. C. Pinho and N. Pinto-Neto, A non inflationary model with scale invariant cosmological perturbations, Phys. Rev. D75, 023516 (2007) [hep-th/ 0610205 ]; Gravitational wave background in perfect fluid quantum cosmologies, Phys. Rev. D73, 104017 (2006) 
[gr-qc/ 0605060$]$; Tensor Perturbations in Quantum Cosmological Backgrounds, JCAP 0507, 014 (2005) [hep-th/ 0509232 ]; J. A. de Barros, N. Pinto-Neto and M. A. Sagioro-Leal, The causal interpretation of dust and radiation fluids non-singular quantum cosmologies, Phys. Lett. A241, 229 (1998).

[33] P. R. Holland, The quantum theory of motion (Cambridge University Press, UK, 1997).

[34] M. Bojowald and G. M. Hossain, Cosmological vector modes and quantum gravity effects, Class. Quantum Grav. 24, 4801 (2007) [gr-qc/0709. 0872]; F.C. Mena, D. J. Mulryne, R. Tavakol, Non-linear vector perturbations in a contracting universe, Class. Quantum Grav. 24, 2721 (2007) [gr-qc/ 0702064 ]; E. J. C. Pinho and N. Pinto-Neto, Scalar and vector perturbations in quantum cosmological backgrounds, Phys. Rev. D76, 023506 (2007) [hep-th/ 0610192 ]; T. Battefeld and R. Brandenberger, Vector perturbations in a contracting Universe, Phys. Rev. D70, 121302 (2004) [hep-th/0406180].

[35] N. Pinto-Neto, Large classical universes emerging from quantum cosmology, Phys. Rev. D79, 083514 (2009) [arXiv:gr/qc/0904.4454]. 